\title{
Predicting Hospitalization Risk of Suspected COVID-19 Patients Using a Machine Learning Methodology
}

\author{
Alesson Mansur Torres \\ Instituto de Matemática e Estatística \\ UERJ \\ Rio de Janeiro, Brazil \\ alesson.mansur@pos.ime.uerj.br
}

\author{
Amanda de Araujo Batista da Silva \\ Departamento de Engenharia Industrial \\ PUC-RIO \\ Rio de Janeiro, Brazil \\ a.mandabatista@hotmail.com
}

\author{
Karla Figueiredo \\ Instituto de Matemática e Estatística \\ UERJ \\ Rio de Janeiro, Brazil \\ karlafigueiredo@ime.uerj.br
}

\begin{abstract}
New SARS-CoV-2 variants and the delay in immunization hinders COVID-19 mitigation. The pressure on healthcare systems caused by the disease has been a challenge for hospital managers. Thus, rapid assessment of hospitalization risk can support the management of hospital resources, increasing treatment opportunities for patients at higher risk, especially in case of huge demands for hospitalizations. In this retrospective study, we propose a hospitalization prediction model based on classical Machine Learning algorithms: Logistic Regression (LR), k-Nearest Neighbors (KNN), Decision Tree (DT), and Support Vector Classifier (SVC). Our proposed classifier combines the results of the two algorithms, which have the best accuracies in order to reduce true-positive misclassification. We included 6, 967 COVID-19 patients from São Paulo, Brazil, who had been admitted at the Hospital Sírio Libanês from February $2^{\text {th }}$ to December $28^{\text {th }}, 2020$. The achieved accuracy was $90.4 \%$, with only $7 \%$ of false-negative patients $(\mathrm{NPV}=0.82)$. Our results suggest that the prediction model proposed can be an efficient tool in screening COVID-19 hospitalization risk patients.
\end{abstract}

Index Terms-COVID-19 Diagnosis, Machine Learning, Hospitalization Risk.

\section{INTRODUCTION}

Although there are a number of COVID-19 vaccines being produced, distributed, and administered worldwide, many regions still suffer high levels of transmission [1]. The lack of controlling virus transmission raises hospital admissions. In low and middle-income countries, where the healthcare systems are frequently strained, the burden is even worse [2]. In addition, the rise of new variants linked to the delay in immunization of the entire population hinders disease mitigation.

Brazil is currently the country with the third-largest number of COVID-19 cases. Still, it surpasses the first and second places regarding the number of deaths per million inhabitants [3]. These numbers demonstrate the need for a better-prepared healthcare system. Furthermore, rapid identification of the risk of hospitalization allows providing more resources and adequate treatment for the most severe patients.

Previous studies have shown promising results when applying machine learning algorithms to predict hospitalization [4], [5]. Blankers, van der Post and Dekker [4] found Gradient
Boosting to be the best performing algorithm (Area Under the Curve (AUC)=0.774) including 39 variables related to patients' socio-demographics, clinical characteristics, and previous mental health care. Wong et al. [5] found Generalized Additive Models (GAM) to be the best model (AUC = $0.70,95 \%$ confidence interval $=0.62-0.79$ ) for hospitalization prediction, including nutritional and inflammation indicators, demographics, comorbidities, and slopes all continuous variables over 6 months as model predictors. In addition, radiological findings at hospital admission are also good predictors of Intensive Care Unit (ICU) admission, as in [6].

Jehi et al. [7] found an AUC of 0.900 (95\% confidence interval of $0.886-0.914)$ in the development cohort and $0.813(0.786,0.839)$ in the validation cohort applying a least absolute shrinkage and selection operator (LASSO) logistic regression algorithm and using statistics of demographic, exposure, clinical, laboratory, social characteristics, and medication history of COVID-19 positive patients who were hospitalized as predictors. Similar to other studies that determine hospitalization risk [7], the rate increased for older people. However, the dataset presented has a low proportion of young patients, suggesting a possible underestimation of the risk found in this population.

We aim to develop a classification model to predict if a COVID-19 suspect patient will be attended to a hospital within two days after realizing a COVID-19 test. We use demographics and clinical characteristics applying an ensemble machine learning methodology. Our model presents reliable results with less complexity than the ones described above. This study can assist physicians in the rapid identification of COVID19 infected patients at high risk of hospitalization, avoiding further harm.

The paper is organized as follows. Section II-A presents the study design. Section II-B presents the data acquisition and the data pre-processing steps. Section II-C presents the Machine Learning methods used to solve the proposed problem and the grid search optimization parameters for the algorithms. Section III presents the results achieved by the proposed ensemble model. Finally, Section IV discusses the results and presents 
the conclusions and perspectives of future works.

\section{MATERIALS AND METHODS}

\section{A. Study design and population}

We performed a retrospective study of patients who attended the Hospital Sírio Libanês (São Paulo, Brazil) with suspicion of COVID-19 infection from February $26^{\text {th }}$ to December $28^{t h}, 2020$. The hospital is an international reference center in healthcare, providing treatment for more than 120,000 patients annually [8]. We only included adult patients that lived in São Paulo state with RT-PCR-confirmed COVID-19 infection, because children usually have clinical behaviour different from adults [9].

\section{B. Data collection}

The data was obtained from the brazilian repository COVID-19 Data Sharing/BR, available at https://repositoriodatasharingfapesp.uspdigital.usp.br/ [10]. The database comprised anonymized registers with features of the clinical spectrum related to the evolution of each COVID-19 patient. It contains demographic data, such as age and gender, and results of laboratory exams regarding blood, vital signs, and hormonal tests.

1) Outcomes and variables: Our main interest was the hospitalization risk, that is, whether a patient should be admitted or not anytime within two days of a positive COVID-19 test. Whereas the prioritization of the first attendance to this patient profile allows for the most appropriate use of resources and physician dedication on the most severe cases. Our goal was to identify clinical indications and individual factors associated with the need for hospitalization. Hence, we collected and assessed participant demographics (age, gender) and clinical characteristics of each patient.

2) Dataset: Since we concentrated the study in people from São Paulo, of the 8,971 COVID-19-suspected patients who attended the hospital, 1,731 were excluded because they lived outside of São Paulo state, and 273 were excluded because they were younger than 18 years old. Thus, 6,967 patients were included in the study (Fig. 1).

Of the 6,967 included in the study, 501 were hospitalized and 5,713 were not hospitalized. The negative class has the greatest number of registers, and we noticed that most of them present clinical characteristics not observed in hospitalized patients. Aiming to select the most important features, we investigated the recurrence of clinical characteristics among the hospitalization risk cases. It is expected that the most recurrent clinical tests are the best predictors. So we evaluated the absolute frequency of each laboratory examination and set a threshold: all the tests whose absolute frequency was less than 400 were excluded, resulting in 35 clinical characteristics. Among the 501 patients with hospitalization risk, 39 patients were excluded because their clinical characteristics were not in the 35 previously selected, and 4 patients were excluded because their clinical characteristics were outliers. Then, the number of patients with hospitalization risk included in the study is 458 .

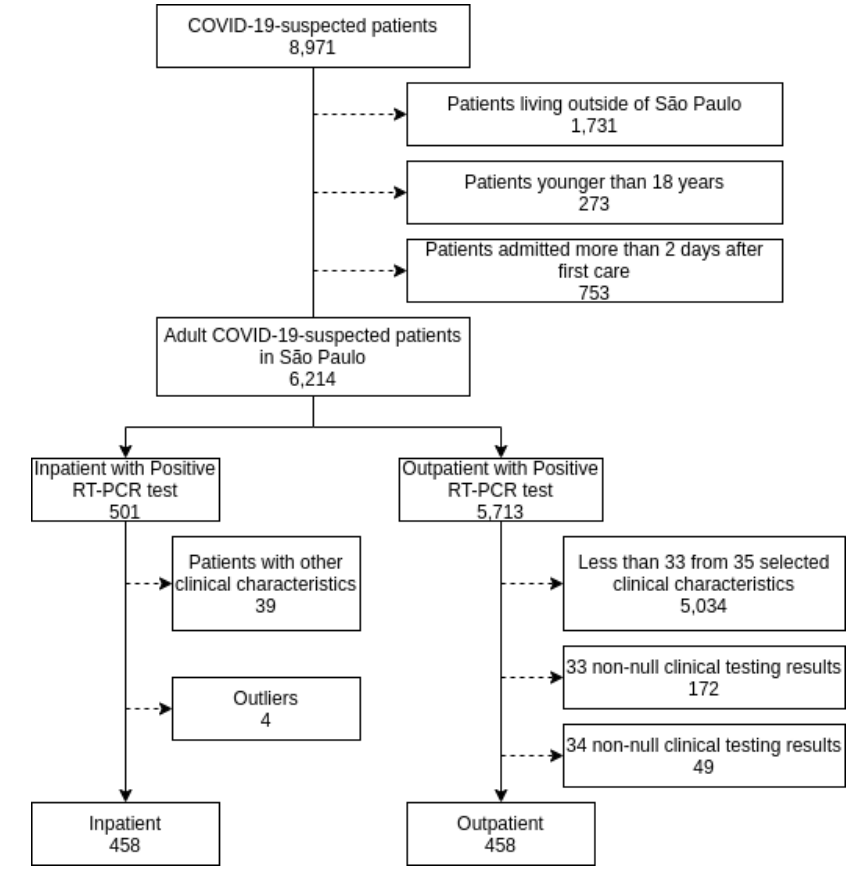

Fig. 1. Inclusion and exclusion criteria flowchart.

TABLE I

SELECTED VARIABLES FOR THE PROBLEM.

\begin{tabular}{|cc|}
\hline & Variables \\
\hline Age & MCH \\
Morphology, SVE & MDRD (afro-descendant-adjusted) \\
Sex & Hematocrit \\
Neutrophils & CKD-EPI (non-African descent-adjusted) \\
Creatinine & Hemoglobin \\
Neutrophils (\%) & MDRD (non-African descent-adjusted) \\
Basophils & Leukocytes \\
Platelets & MPV \\
Basophils (\%) & Lymphocytes \\
MCV & Potassium \\
MCHC & Lymphocytes (\%) \\
RDW & Sodium \\
Eosinophils & Monocytes \\
C-reactive protein & ALT \\
Eosinophils (\%) & Monocytes (\%) \\
Urea & AST \\
Erythrocytes & Morphology, SB \\
CKD-EPI (afro-descendant-adjusted) & Fibrin Dimers \\
\hline
\end{tabular}

Of the 5,713 patients who were not admitted to the hospital, 5,034 were excluded because less than 33 from 35 clinical characteristics were not null. The remaining 679 patients were sorted by a clinical characteristic ranking that sorted them from the one with fewer null test results to the one with more null test results. It resulted in 432 patients with 35 non-null clinical testing results, 75 with 34 non-null clinical testing results, and 172 with 33 non-null clinical testing results. So, in order to create a balanced database and avoid bias from missing values, the 432 patients with 35 non-null results were joined with 26 from the 75 patients with 34 non-null results. The variables selected (clinical and demographic characteristics) 
are available in Table I.

The final dataset comprises 916 patients, whereby 458 are classified as patients with hospitalization risk and 458 are classified as patients with no hospitalization risk. The missing values were replaced with an average value based on the laboratory examination references. It was supposed that missing results for clinical characteristics means that the physicians do not expect nonstandard results. The final database was shuffled and split into two: 738 registries $(80 \%)$ for training/validation and $178(20 \%)$ for testing. All the features were normalized to a standard normal distribution [11].

\section{Machine Learning Algorithms}

We assessed the hospitalization risk in COVID-19 patients using four different machine learning algorithms: Logistic Regression (LR) [12], K-Nearest Neighbors (KNN) [13], Decision Tree (DT) [13], and Support Vector Classifier (SVC) [12]. The models were implemented with the open-source package Scikit-learn provided for Python programming language. The evaluation metrics include accuracy, area under the ROC curve (AUC), Specificity, Sensitivity, F1 score, PPV and NPV. The ReliefF algorithm [14] was employed in order to investigate the most important features in the problem. II-C1, II-C2, II-C3, II-C4 and II-C5 will briefly discuss the employed models and the feature selection algorithm.

1) Logistic Regression: In spite of the name, Logistic Regression (LR) is a linear model for classification rather than regression. Considering a data set where the targets $Y$ falls into the categories 1 or 0 , the Logistic Regression models the classposterior probability that $Y$ belongs to a particular category instead of modeling the response $Y$ directly. In other words, Logistic Regression models the probability $p$ of a sample $x$ belonging to a class $y$ by the relationship $p(x)=P(y \mid x)$. The probabilities that describe the possible outcomes of a single trial are modeled using a logistic function, also known as the Sigmoid function [12].

2) k-Nearest Neighbors: The K-Nearest Neighbors (KNN) classification algorithm is a type of instance-based learning that is memory-based and requires no model to be fit [13]. Given an instance (or query point) $x_{0}$, the algorithm finds the $k$ training points $x_{(r)}, r=1, \ldots, k$ closest in distance to $x_{0}$. Then $x_{0}$ is classified by the majority vote among the $k$ nearest neighbors. In other words, $x_{0}$ is assigned to the majority data class within their nearest neighbors [13]. $k$ is an integer value specified by the user.

3) Decision Tree: Decision Trees (DT) are supervised learning methods applied in both regression and classification. One of the most popular algorithms is called CART (Classification And Regression Trees) and it builds a decision tree that recursively partitions the feature space such that the samples with the same labels or similar target values are grouped. The CART algorithm splits the database into several nodes and, in order to evaluate the quality of a particular split, either the Gini index or the Cross-entropy are employed [13]. This quality is usually called node impurity and it measures the proportion of different class observations in the node. When the impurity value is zero (or the lowest as possible) a leaf node is reached. The majority class of the observations classifies the samples assigned to a node in the node [12].

4) Support Vector Classifier: When it is possible to construct a hyperplane that separates the training observations perfectly according to their class labels, it is possible to construct a very natural classifier: a test observation will be assigned to a class depending on which side of the hyperplane it is located [12]. However, when the data cannot be perfectly separated using a hyperplane, it is desired to find a classifier that does not perfectly separate the two classes: it can misclassify a few training observations in order to do a better job in classifying the remaining observations. The Support Vector Classifier (SVC), also called soft margin classifier, does this, allowing some observations to be on the incorrect side of the margin, or even the incorrect side of the hyperplane. Since SVC classifies a test observation depending on which side of a hyperplane it lies, observations on the wrong side of the hyperplane correspond to training observations that will be misclassified. Thus, it prevents overfitting the training data and provides greater robustness to individual observations [12].

5) ReliefF: According to [14], the ReliefF (or Relief-F) algorithm is not limited to two-class problems, is more robust, and can deal with incomplete and noisy data. ReliefF randomly selects an instance $R_{i}$, then searches $k$ of its nearest neighbors from the same class, called nearest hits $H_{j}$, and also $k$ nearest neighbors from each of the different classes, called nearest misses $M_{j}(C)$. It updates the quality estimation $W[A]$ for all attributes $A$ depending on their values for $R_{i}$, hits $H_{j}$, and misses $M_{j}(C)$. If instances $R_{i}$ and $H_{j}$ have different values of the attribute $A$ then the attribute $A$ separates two instances with the same class which is not desirable so the quality estimation $W[A]$ is decreased. On the other hand, if instances $R_{i}$ and $M_{j}(C)$ have different values of attribute $A$ then attribute $A$ separates two instances with different class values which are desirable so the quality estimation $W[A]$ is increased. The whole process is repeated $m$ times, where $m$ is a user-defined parameter. This update formula is similar to that of Relief, except that the contribution of all the hits and all the misses are averaged. Selection of $k$ hits and misses is the basic difference to Relief and ensures greater robustness of the algorithm concerning noise. User-defined parameter $k$ controls the locality of the estimates.

\section{Methodology}

1) Proposed Approach: Aiming to reduce the misclassifications of high hospitalization risk patients, we propose a classifier that combines the results of the two models that achieve the best performances. Unless the two models categorize the sample as no hospitalization risk, our proposed model will assign it to a hospitalization risk class. This approach can clearly increase the false-positive rate. However, it is a safe way to treat the cases whose clinical characteristics suggest a positive class sample.

2) Parameters: We employed the grid search approach with standard 10-fold Cross-Validation on the training and 
validation set for adjusting hyper-parameters. The evaluated grid search parameters for the four employed models are presented in Tables II, III, IV and V.

TABLE II

GRID SEARCH PARAMETERS FOR LR.

\begin{tabular}{|c|c|}
\hline Param & Values \\
\hline penalty & '11', '12', 'elasticnet', 'none' \\
\hline dual & True, False \\
\hline $\mathrm{C}$ & $.5, .6, .7, .8, .9,1.0,1.2,1.4,1.6,1.7,1.8,1.9,2.0$ \\
\hline fit_intercept & True, False \\
\hline intercept_scaling & $1.0,1.5,2.0,2.5,3.0,3.5,4.0,5.0,6.0,8.0,10.0$ \\
\hline random_state & None, 42 \\
\hline solver & 'newton-cg', 'lbfgs', 'liblinear', 'sag', 'saga' \\
\hline
\end{tabular}

TABLE III

GRID SEARCH PARAMETERS FOR KNN.

\begin{tabular}{|c|c|}
\hline Param & Values \\
\hline n_neighbors & $2,3,4,5,6,7,8,9,10$ \\
\hline weights & 'uniform', 'distance' \\
\hline algorithm & 'ball_tree', 'kd_tree', 'brute' \\
\hline leaf_size & $15,20,25,30,40,50$ \\
\hline p & 1,2 \\
\hline
\end{tabular}

TABLE IV

GRID SEARCH PARAMETERS FOR DT

\begin{tabular}{|c|c|}
\hline Param & Values \\
\hline max_depth & $2,3,4,5,6,7,8,9,10$ \\
\hline max_features & $2,3,4,5,6$ \\
\hline min_samples_split & $5,6,8,10,15,20,25,30,40,50$ \\
\hline min_samples_leaf & $2,5,8,10,12,15,20$ \\
\hline
\end{tabular}

TABLE V

GRID SEARCH PARAMETERS FOR SVC.

\begin{tabular}{|c|c|}
\hline Param & Values \\
\hline $\mathrm{C}$ & $.5, .6, .7, .8, .9,1.0,1.2,1.4,1.6,1.7,1.8,1.9,2.0$ \\
\hline kernel & 'linear', 'poly', 'rbf', 'sigmoid' \\
\hline degree & $1,2,3,4,5,6$ \\
\hline gamma & 'scale','auto' \\
\hline coef0 & $0.0,0.1,0.2,0.3,0.4,0.5,0.6,0.7,0.8,0.9$ \\
\hline
\end{tabular}

\section{RESULTS}

Tables VI and VII show the parameters related to the best cross-validation performances obtained with each algorithm. The overall validation accuracies obtained for each model using the parameters described in Tables VI and VII are presented in Table VIII. LR presented better results than other classifiers, which suggests a good linear relationship between the features and the output.

Since LR achieved the best overall accuracy value, considering the mean value of validation (cross-validation), it was chosen as the first model to be employed on the test data set. Fig. 2 shows the confusion matrix for the LR model on the test set. This model obtained a classification accuracy
TABLE VI

BEST PARAMETERS FOR LR AND KNN MODELS.

\begin{tabular}{|c|c|}
\hline LR & KNN \\
\hline dual=False & algorithm='ball_tree' \\
\hline fit_intercept=True & leaf_size=15 \\
\hline intercept_scaling=1.0 & n_neighbors=8 \\
\hline penalty='none' & $\mathrm{p}=1$ \\
\hline random_state=None & weights='uniform' \\
\hline solver='newton-cg' & \\
\hline
\end{tabular}

TABLE VII

BEST PARAMETERS FOR DT AND SVC MODELS.

\begin{tabular}{|c|c|}
\hline DT & SVC \\
\hline max_depth=10 & $\mathrm{C}=1.7$ \\
\hline max_features=5 & coef0 $=0.9$ \\
\hline min_samples_leaf=10 & degree=3 \\
\hline min_samples_split=25 & gamma='scale' \\
\hline
\end{tabular}

of $89.3 \%$ and an AUC of 0.97. However, there is a large false-negative rate $(18 \%)$ representing a misclassification of higher hospitalization risk patients. Due to the nature of the problem, the hospitalization risk should be as low as possible. Otherwise, it can lead to inappropriate treatments and aggravate the patient's condition.

Therefore, aiming to assess the trade-off between false positives and false negatives, we employed the SVC model on the test data as a second classification model. This model achieved the second-best overall accuracy on training and validation data sets (Table VIII). The obtained results for the LR and SVC models evaluation metrics are presented in Table IX. Fig. 3 shows the confusion matrix for the SVC model.

Although the overall training and validation accuracy of the LR model is greater than the accuracies achieved by the other three models, it is not much bigger than the one achieved by the SVC model. An interesting result is that the test classification accuracy of the SVC model is better than the one achieved by the LR model. On the other hand, the LR AUC is greater than the SVC AUC (Fig. 4).

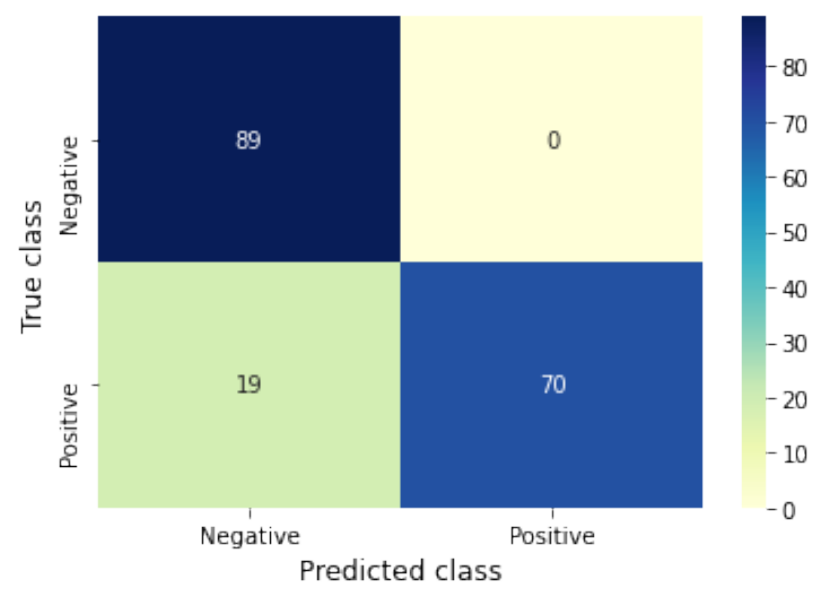

Fig. 2. Confusion Matrix of LR model. 
TABLE VIII

MODEL'S ACCURACIES FOR TRAINING AND VALIDATION DATASETS

\begin{tabular}{|c|c|c|}
\hline Model & Train (\%) & Validation (\%) \\
\hline LR & 98.2 & 95.5 \\
\hline KNN & 90.0 & 87.0 \\
\hline DT & 85.9 & 78.7 \\
\hline SVC & 97.2 & 93.7 \\
\hline
\end{tabular}

TABLE IX

EVALUATION METRICS FOR LR, SVC AND PROPOSED MODELS FOR TEST SET.

\begin{tabular}{|c|c|c|c|}
\hline & \multicolumn{3}{|c|}{ Classifier } \\
\hline & LR & SVC & Proposed \\
\hline Accuracy & 0.893 & 0.904 & 0.904 \\
\hline AUC & 0.974 & 0.959 & 0.959 \\
\hline Specificity & 0.824 & 0.924 & 0.924 \\
\hline Sensitivity & 1.000 & 0.883 & 0.883 \\
\hline F1 score & 0.88 & 0.91 & 0.91 \\
\hline PPV & 0.787 & 0.933 & 0.933 \\
\hline NPV & 0.824 & 0.929 & 0.929 \\
\hline
\end{tabular}

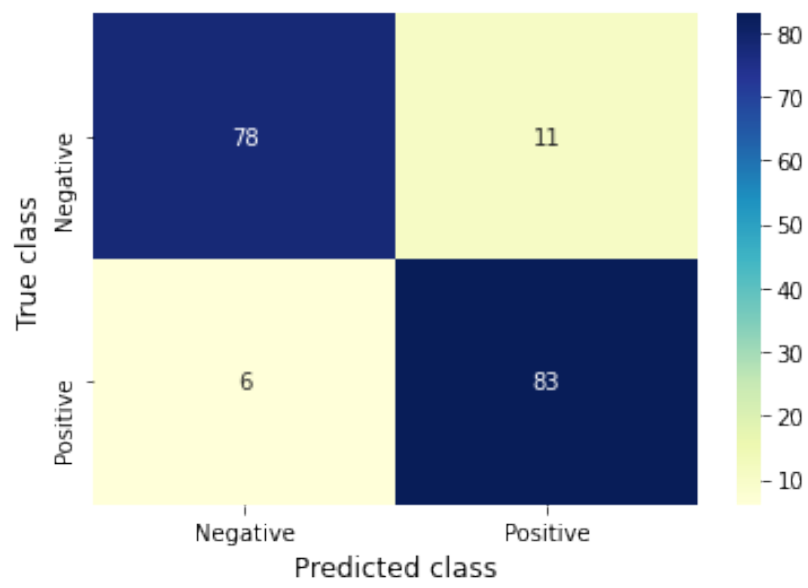

Fig. 3. Confusion Matrix of SVC model

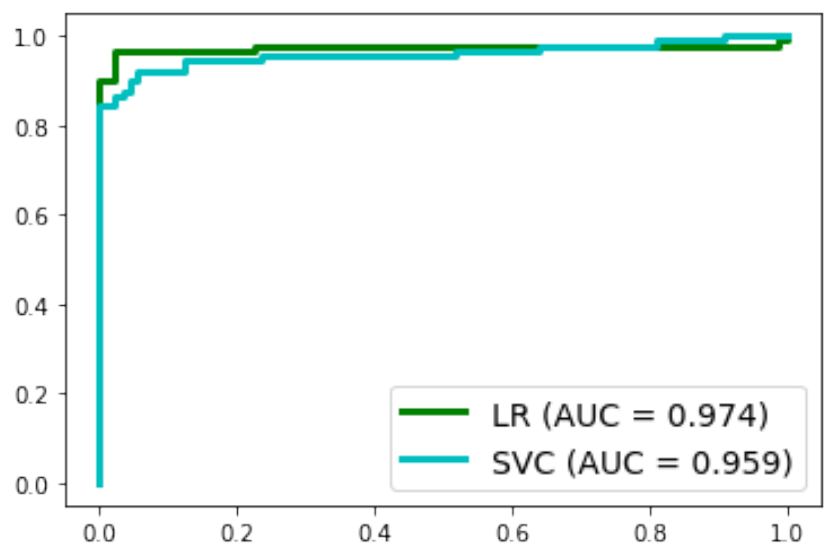

Fig. 4. ROC curve and AUC for LR and SVC models
Our proposed classifier integrates the two results by the following rule: whenever a model's result indicates hospitalization risk, it is treated as hospitalization risk, regardless of the model of origin. It is an approach that aims to reduce the true-negative misclassifications. The confusion matrix for the proposed classifier is presented in Fig. 5.

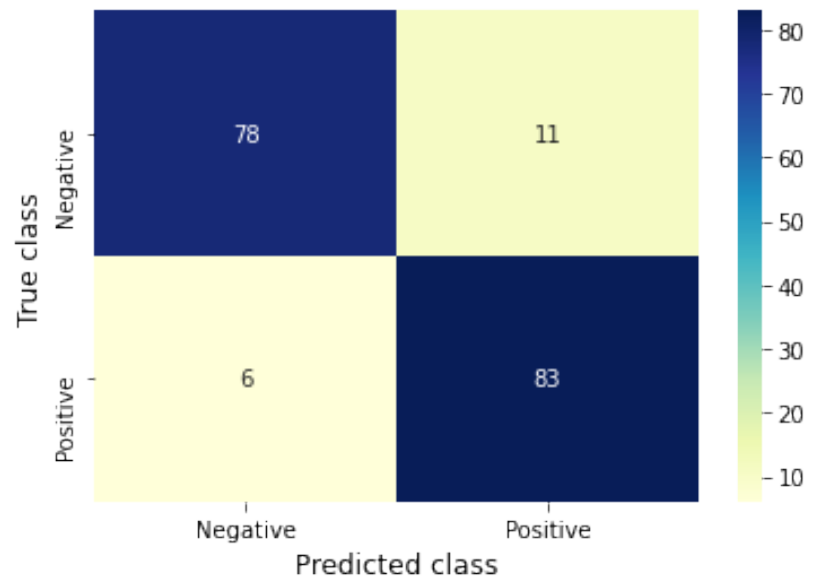

Fig. 5. Confusion Matrix of the proposed model for test set

As one can see, the confusion matrix presented in Fig. 5 is the same obtained by the SVC model (Fig. 3). It is an expected result because since LR performed more false positives than SVC, our proposed model will classify the samples labeled as negative by LR but positive by SVC as positive. However, on the other hand, since SVC performed more false negatives than LR, these samples will be labeled as positive by our model as well.

The performance metrics' values presented in Table IX indicate that the choice of the proposed model as the hospitalization risk predictor is aligned with our expectations. Our proposed model presented the same positive predictive value (PPV) and negative predictive value (NPV) metrics as the SVC model, which means that it has at least the same performance as obtained by SVC on testing (Fig. 3 and Fig. 5). Compared with the LR model, the misclassification of the positive class (hospitalization risk) is lower in ours than in LR. Therefore, concerning the true positive and true negative prediction rates, our model has a lower misclassification rate as SVC, which means, this model is preferable as it avoids the misclassification of a patient with higher hospitalization risk rates

We applied a feature selection strategy to improve the overall accuracy of the method. The algorithm used was the ReliefF. It returns a feature importance rank by sorting the feature matrix columns from the most important feature to the less important feature. Contrary to our expectations, the algorithm indicated leukocytes (responsible for maintaining the immune system) as the worst predictor attribute. Even so, we followed this recommendation, and this attribute was removed. The accuracy reduced to $61.8 \%$, confirming the expectation mentioned above. Therefore, for this reason, all 
attributes were kept, and we concluded that the feature selection approach using the ReliefF algorithm did not result in any improvements.

\section{CONCLUSion}

We developed models that can predict the hospitalization risk among COVID-19 suspect patients. Our prognostic model could help clinical decision-makers in resource allocation, designating order for exams, and prioritizing patients with higher hospitalization risk. We analyzed the main predictors (demographics, vital signs, and laboratory results) associated with the hospitalization risk in COVID-19 suspect patients. Our approach allows the identification of patients with higher chances of hospitalization and guides decision-makers in better resource allocation. We applied four different machine learning models and we chose the LR and the SVC model as the base of our proposed classifier. Any other models in pairs would have resulted in lower performance because the other models presented lower accuracy.

According to these findings, the hospitalization risk increased for patients recently diagnosed with COVID-19 who are older, black, male, have comorbidities and low economic status [7], [15]. Our models considered age and sex, other variables could not be accessed due to the lack of information.

Our proposed model performed an accuracy of $90.4 \%$, an AUC of 0.959 , a PPV of 0.933 and a NPV of 0.929 including 35 features. This result is better than the one found by [4], that found an $\mathrm{AUC}=0.774$ including 39 features, and the one found by [5], that found an $\mathrm{AUC}=0.7$. Our result is also better than the one found by [7], that found an $\mathrm{AUC}=0.813$ in the validation cohort using 48 features.

Despite the good results, the following questions should be considered: 1) we could not include patients not infected by COVID-19 due to the lack of data; 2) there is no information about which department the patient was admitted (ward or ICU), making it impossible to know the case severity and the level of attention and resources intended for the patient; and 3) the registers do not indicate timing, resulting in inaccuracy in the chronological order of the results. There might also be selection bias because private hospitals present particularities not common to other establishments, specially compared to public hospitals.

For future works, we suggest assessing the hospitalization risk, also considering non-infected patients, and applying the models in datasets from other hospitals, which can provide information not available in the dataset used such as radiologic data.

\section{REFERENCES}

[1] Sah, P. et al. Accelerated vaccine rollout is imperative to mitigate highly transmissible COVID-19 variants, EClinicalMedicine, vol. 35, May 2021, doi: 10.1016/j.eclinm.2021.100865.

[2] Okereke, M. et al. Impact of COVID-19 on access to healthcare in low- and middle-income countries: Current evidence and future recommendations, Int. J. Health Plann. Manage., vol. 36, no. 1, pp. 13-17, 2021, doi: 10.1002/hpm.3067.

[3] Coronavirus Update (Live), [cited 13 Jun 2020]. Available: https://www.worldometers.info/coronavirus/\#countries
[4] Blankers, M., van der Post, L. F. M. and Dekker, J. J. M. Predicting hospitalization following psychiatric crisis care using machine learning., BMC Med. Inform. Decis. Mak., vol. 20, no. 1, p. 332, Dec. 2020, doi: 10.1186/s12911-020-01361-1.

[5] Wong, M. M. Y. et al. Prediction of Mortality and Hospitalization Risk Using Nutritional Indicators and Their Changes Over Time in a Large Prevalent Hemodialysis Cohort, J. Ren. Nutr. Off. J. Counc. Ren. Nutr. Natl. Kidney Found., vol. 30, no. 1, pp. 69-78, Jan. 2020, doi: 10.1053/j.jrn.2019.01.013.

[6] García-Menaya, J. M. et al. Outcomes and Laboratory and Clinical Findings of Asthma and Allergic Patients Admitted With Covid-19 in a Spanish University Hospital., Frontiers in Pharmacology, vol. 11, p. 1505, Sep. 2020, doi: 10.3389/fphar.2020.570721.

[7] Jehi, L. et al. Development and validation of a model for individualized prediction of hospitalization risk in 4,536 patients with COVID-19, PLOS ONE, vol. 15, no. 8, p. e0237419, Aug. 2020, doi: 10.1371/journal.pone.0237419.

[8] Hospital Sírio Libanês, [cited 13 Jun 2020]. Available: https://www.hospitalsiriolibanes.org.br/institucional/Paginas/default.aspx

[9] Bearer, C. F. How are children different from adults?, Environmental Health Perspectives, 103(Suppl 6):7-12, 1995.

[10] Mello, L. E. et al. 2020. Opening Brazilian COVID-19 patient data to support world research on pandemics., Zenodo 20(30 July).

[11] Morettin, P. A. and Bussab, W. O. Estatística Básica. 5Th Edition. Saraiva, 2004

[12] James, G., Witten, D., Hastie, T. and Tibshirani, R. An Introduction to Statistical Learning with Applications in R. In Springer Texts in Statistics. Springer, 2013. ISSN: 1431-875X

[13] Hastie, T., Tibshirani, R. and Friedman, J. The Elements of Statistical Learning: Data Mining, Inference, and Prediction. Second Edition. In Springer Series in Statistics. Springer, 2017.

[14] Robnik-Šikonja, M., Kononenko, I. Theoretical and Empirical Analysis of ReliefF and RReliefF., Machine Learning 53, 23-69 (2003). https://doi.org/10.1023/A:1025667309714

[15] S. Wollenstein-Betech, A. A. B. Silva, J. L. Fleck, C. G. Cassandras, and I. C. Paschalidis Physiological and socioeconomic characteristics predict COVID-19 mortality and resource utilization in Brazil, PloS One, vol. 15 , no. 10 , p. e0240346, 2020, doi: 10.1371/journal.pone.0240346 